\title{
SPARSE SPECTRAL FACTORIZATION: UNICITY AND RECONSTRUCTION ALGORITHMS
}

\author{
Yue M. Lu ${ }^{(a)}$ and Martin Vetterli ${ }^{(b)}$

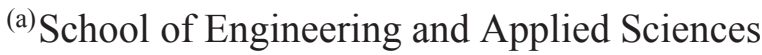 \\ Harvard University, Cambridge, MA 02138, USA \\ (b) School of Computer and Communication Sciences \\ Ecole Polytechnique Fédérale de Lausanne (EPFL), Lausanne, Switzerland \\ Email: yuelu@seas.harvard.edu; martin.vetterli@epfl.ch
}

\begin{abstract}
Spectral factorization is a classical tool in signal processing and communications. It also plays a critical role in X-ray crystallography, in the context of phase retrieval. In this work, we study the problem of sparse spectral factorization, aiming to recover a one-dimensional sparse signal from its autocorrelation. We present a sufficient condition for the recovery to be unique, and propose an iterative algorithm that can obtain the original signal (up to a sign change, time-shift and time-reversal). Numerical simulations verify the effectiveness of the proposed algorithm.
\end{abstract}

Index Terms - Phase retrieval, sparse spectral factorization, compressed sensing, frame reconstruction without phase

\section{INTRODUCTION}

Spectral factorization is a widely used tool in signal processing, communications, optimal control, and many other disciplines. Let $x_{n}$ be a finite-length real-valued sequence, and $a_{n}$ its autocorrelation, i.e.,

$$
a_{n} \stackrel{\text { def }}{=} \sum_{k} x_{k} x_{k-n}=\left(x_{k} * x_{-k}\right)_{n} .
$$

The goal of spectral factorization is to recover $x_{n}$ from $a_{n}$. Representing (1) in the Fourier domain, we get

$$
A\left(e^{j \omega}\right)=X\left(e^{j \omega}\right) X^{*}\left(e^{j \omega}\right)=\left|X\left(e^{j \omega}\right)\right|^{2},
$$

where $A\left(e^{j \omega}\right)$ and $X\left(e^{j \omega}\right)$ are the Fourier transforms of $a_{n}$ and $x_{n}$, respectively. It follows that the task of spectral factorization is equivalent to recovering the missing phase information of $X\left(e^{j \omega}\right)$ from its squared magnitude $A\left(e^{j \omega}\right)$. This problem is often called phase retrieval [1] in the literature, and plays a critical role in fields such as $\mathrm{X}$-ray crystallography and astronomical imaging.

Spectral factorization and phase retrieval have been extensively studied in the past (see, e.g., $[1,2]$ for comprehensive surveys). One important question is that of unicity, i.e., whether the sequence $x_{n}$ can be uniquely determined by its autocorrelation $a_{n}$. The answer is negative in the one-dimensional (1-D) case [3]. To see this, we can rewrite (1) in the $z$-domain as $A(z)=X(z) X\left(z^{-1}\right)$.

By construction, the roots of the Laurent polynomial $A(z)$ must appear in pairs: $u$ is a root of $A(z)$ if and only if $u^{-1}$ is a root. By further limiting our attention to real-valued signal $x_{n}$, the roots of $A(z)$ will in general appear in quadruples:

$$
u, u^{*}, u^{-1}, u^{-*},
$$

This work was supported by an ERC Advanced Grant - Support for Frontier Research - SPARSAM Nr: 247006. which might "collapse" into a pair or even a single point when $u$ lies on the unit circle. Spectral factorization boils down to distributing these roots between $X(z)$ and $X\left(z^{-1}\right)$. To be clear, the assignment is nonunique. For each quadruple of roots in (2), we can either let $\left(u, u^{*}\right) \rightarrow X(z)$ and $\left(u^{-1}, u^{-*}\right) \rightarrow X\left(z^{-1}\right)$, or have $\left(u^{-1}, u^{-*}\right) \rightarrow X(z)$ and $\left(u, u^{*}\right) \rightarrow X\left(z^{-1}\right)$.

Either choice leads to a valid but different $X(z)$. When the signal $x_{n}$ is of length $N$, the total number of different factorizations is exponential in $N$. In classical spectral factorization [2], one avoids this ambiguity by looking for the so-called "minimum phase" solution, assigning all roots that are inside the unique circle to $X(z)$.

In this work, we consider the following problem, which we call sparse spectral factorization. Let $x_{n}$ be a 1-D real-valued signal of length $N$, containing only $K \ll N$ nonzero elements. Can we reconstruct $x_{n}$ from its autocorrelation $a_{n}$ ? Intuitively, although there exist inherent ambiguities in assigning the roots of $A(z)$, the sparsity constraint of the original signal $x_{n}$ will greatly limit the possible choices, hopefully leading to unique solutions. Sparsity is a reasonable assumption for the underlying signals in many applications (e.g., the unknown channel in a multi-path environment, or the electron density of protein crystals). It is therefore interesting to investigate if this additional prior information can be incorporated to an advantage.

In an early paper on phase retrieval [4], Crimmins and Fienup showed that functions with sufficiently separated supports (therefore, sparse in some sense) can be uniquely determined by their Fourier magnitudes. Their study focuses on continuous-domain functions and assumes that the supports are known a priori. Here, we investigate the discrete case, under the more challenging setting of unknown supports, and provide concrete algorithms for signal reconstructions. The sparsity prior has also been considered in a recent work [5] to derive efficient acquisition schemes for phase retrieval. In general, the autocorrelation of a $K$-sparse signal is also sparse, consisting of up to $K^{2}$ nonzero elements, and thus can be determined from $\mathcal{O}\left(K^{2} \log (N)\right)$ Fourier samples [5]. Once the autocorrelation is obtained, the actual spectral factorization (or phase retrieval) in that work is still done by using classical algorithms [1].

In this paper, we present the following contributions:

1. Unicity: We show in Section 2 that there always exist sparse signals that cannot be determined by their autocorrelations, no matter how sparse they are. However, when the sparsity patten is nonuniform, most signals are indeed recoverable. We present a sufficient condition (Theorem 1) for the recovery to be unique.

2. Reconstruction algorithm: Our proof of Theorem 1 is constructive in nature, and directly leads to a concrete reconstruction algorithm. In Section 3, we present an iterative scheme based on singular value projections that can efficiently recover a sparse signal from its autocorrelation. 


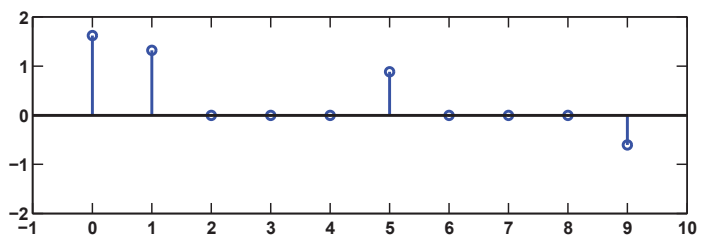

(a) $x_{n}$

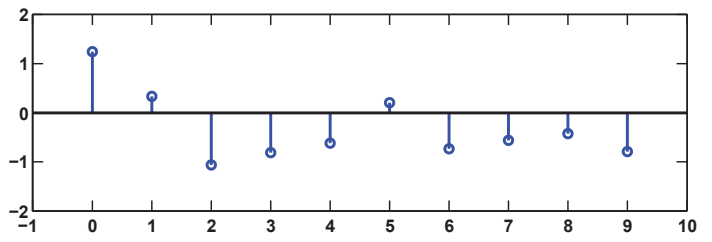

(b) $\widehat{x}_{n}$

Fig. 1. Example of how the sparsity prior can help make spectral factorization unique. (a) A sparse signal $x_{n}$, supported on $[0,9]$ and containing 4 nonzero coefficients. (b) A different signal $\widehat{x}_{n}$, having the same autocorrelation as $x_{n}$. In the $z$-domain, $X(z)$ and $\widehat{X}(z)$ differ only in one root, but $\widehat{x}_{n}$ has completely lost the sparsity of $x_{n}$.

\section{ON THE UNICITY OF SPARSE SPECTRAL FACTORIZATION}

\subsection{Preliminaries}

Before presenting our results on the unicity problem, we first need to precisely define what we mean by "unique" reconstruction. For any real-valued $K$-sparse signal $x_{n}$, the following variations

$$
-x_{n}, \quad x_{n-m}, \quad x_{-n}
$$

are still $K$-sparse and have exactly the same autocorrelation. Therefore, the best we can strive for is to reconstruct $x_{n}$ up to a sign change, an unknown shift (by $m$ ), and a time-reversal.

Without loss of generality, we shall assume in what follows that the signal $x_{n}$ is supported on $n=0,1, \ldots, N-1$, with the two "endpoints" $-x_{0}$ and $x_{N-1}-$ being nonzero. This support constraint removes the ambiguity in the relative location of $x_{n}$. In practice, the signal length $N$ can be estimated from the support of $a_{n}$, which is equal to $[-(N-1),(N-1)]$. We can further resolve the unknown sign of $x_{n}$ by setting $x_{0}>0$. Finally, the only remaining ambiguity is a possible time-reversal, i.e., $x_{n}$ versus $x_{N-1-n}$.

Example 1 Consider a sparse signal $x_{n}$ shown in Figure 1(a), with $N=10$ and $K=4$. Its $z$-transform $X(z)$ contains one real-valued root $u=0.7647$ and 4 pairs of complex roots. By "flipping" the real root, i.e., $u \rightarrow u^{-1}$, but keeping all the complex ones, we can obtain a new sequence $\widehat{x}_{n}$ [see Figure 1(b)] which has the same autocorrelation as $x_{n}$. However, comparing Figure 1(a) with Figure 1(b), we see that the incorrect assignment of just one root will make the resulting signal drastically non-sparse.

The above example suggests that the sparsity constraint of $x_{n}$ can indeed help to make the spectral factorization unique. Unfortunately, we can always find sparse signals that cannot be uniquely determined by their autocorrelations. For example, consider the special case where the nonzero elements of $x_{n}$ are located in equal distances. In this case, we can write

$$
x_{n}=\sum_{k=0}^{K-1} c_{k} \delta_{n-k M}
$$

for some interval $M>0$.

Proposition 1 Two uniformly sparse signals $x_{n}=\sum_{k} c_{k} \delta_{n-k M}$ and $y_{n}=\sum_{k} d_{k} \delta_{n-k M}$ have the same autocorrelation if and only if

$$
C(z) C\left(z^{-1}\right)=D(z) D\left(z^{-1}\right),
$$

i.e., the two downsampled sequences $c_{k}$ and $d_{k}$ have the same autocorrelation.

Proof We note that $X(z)=C\left(z^{M}\right)$ and $Y(z)=D\left(z^{M}\right)$. Now, to show the sufficiency part, we assume (4) holds. It follows that

$X(z) X\left(z^{-1}\right)=C\left(z^{M}\right) C\left(z^{-M}\right)=D\left(z^{M}\right) D\left(z^{-M}\right)=Y(z) Y\left(z^{-1}\right)$.

The necessity part is similar. $X(z) X\left(z^{-1}\right)=Y(z) Y\left(z^{-1}\right)$ implies that

$$
C\left(z^{M}\right) C\left(z^{-M}\right)=D\left(z^{M}\right) D\left(z^{-M}\right),
$$

hence, after downsampling by $M$, we get (4).

Remark 1 For signals $x_{n}$ with uniform supports as in (3), if we choose their coefficients $\left\{c_{k}\right\}$ from an i.i.d. Gaussian distribution, we can find (with probability one) different sequences $\left\{d_{k}\right\}$ for which (4) holds. It follows from Proposition 1 that almost all signals with uniform sparsity patterns are "nonrecoverable" from their autocorrelations.

The prospect is much brighter when the sparsity patterns are nonuniform. Numerical simulations with randomly generated coefficients strongly suggest that, when the sparsity patterns are nonuniform, almost all such sequences can be uniquely determined from their autocorrelations.

\subsection{A Sufficient Condition for Unique Reconstruction}

In what follows, we present a sufficient condition for the unicity of sparse spectral factorization (Theorem 1). Our proof is constructive and leads to a concrete algorithm which will be described in Section 3.

For a $K$-sparse signal $x_{n}$, let $\mathbb{1}_{n}$ represent the indicator function of its support set, i.e., $\mathbb{1}_{n}=1$ if $x_{n} \neq 0$ and $\mathbb{1}_{n}=0$ otherwise. Denote by

$$
\mathcal{S} \stackrel{\text { def }}{=} \operatorname{supp} a_{n} \quad \text { and } \quad \widetilde{\mathcal{S}} \stackrel{\text { def }}{=} \operatorname{supp}\left(\mathbb{1}_{k} * \mathbb{1}_{-k}\right)_{n}
$$

the support sets of the autocorrelation sequences of $x_{n}$ and $\mathbb{1}_{n}$, respectively. We consider two conditions in our derivations:

Condition 1 We assume that $\mathcal{S}=\widetilde{\mathcal{S}}$.

By construction, we always have $\mathcal{S} \subseteq \widetilde{\mathcal{S}}$. Here, Condition 1 requires that there should be no "cancellation" of support in the autocorrelation sequence $a_{n}$. This requirement is fairly weak: If the coefficients of the signal $x_{n}$ are to be drawn from an i.i.d. Gaussian distribution, then Condition 1 holds with probability one.

To describe the second condition, we first need to construct a matrix from $\mathcal{S}$. Denote by $L \stackrel{\text { def }}{=}|\mathcal{S}|$ the cardinality of the set $\mathcal{S}$. Let $M \geq 4 N$ be an integer, and $\boldsymbol{F}_{M}$ the discrete Fourier transform (DFT) matrix of size $M \times M$. We build an $M \times L$ matrix $A$ whose columns are picked from $\boldsymbol{F}_{M}$. Specifically, the $n$-th column of $\boldsymbol{F}_{M}$ is chosen if and only if $n \in \mathcal{S}$. Note that here we are using a circular indexing scheme. So when $n$ is a negative number, the $n$th column actually means the $(M-1+n)$ th column. 
Condition 2 Denote by $\mathcal{R}(\boldsymbol{A})$ the range space of $\boldsymbol{A}$. We require that almost all $\boldsymbol{c} \in \mathcal{R}(\boldsymbol{A})$ be uniquely determined (up to a constant phase or complex-conjugation) from their absolute values. Specifically, for almost all $\boldsymbol{c} \in \mathcal{R}(\boldsymbol{A})$, we need

$$
\left\{\boldsymbol{v} \in \mathcal{R}(\boldsymbol{A}):\left|v_{n}\right|=\left|c_{n}\right|\right\}=\left\{\xi \boldsymbol{c}, \xi \boldsymbol{c}^{*}: \text { for }|\xi|=1\right\} .
$$

Readers familiar with frame theory will recognize that the matrix $\boldsymbol{A}$ constructed above corresponds to the analysis operator of a tight DFT frame. The redundancy factor of such a frame is equal to $M / L$. Condition 2 means that the frame expansion coefficients should be uniquely determined by their absolute values. The problem of frame reconstruction from magnitude information was recently studied in [6]. Intuitively, this task is feasible because the loss of phase information can be compensated for by the redundancy of the frame.

Theorem 1 Suppose that Conditions 1 and 2 hold. Then, for almost all $K$-sparse signals $x_{n}$ supported on $\mathbb{1}_{n}$, we can uniquely determine $x_{n}$ (up to a time-reversal) from its autocorrelation sequence $a_{n}$.

Proof Let $M \geq 4 N$ be an integer. Extend the original sequence $x_{n}$ to length- $M$ by inserting zeros from indices $N$ to $M-1$, and call the extended sequence $\widehat{x}_{n}$. Denote by $f_{m}$ the $m$ th DFT coefficient of $\widehat{x}_{n}$. From the autocorrelation $a_{n}$, we have access to $\left\{\left|f_{m}\right|^{2}\right\}_{m}$.

Now, consider the sequence $c_{m} \stackrel{\text { def }}{=} f_{m} f_{m-1}^{*}$, for $0 \leq m<M$. We can show ${ }^{1}$ that the support of the inverse DFT of $c_{m}$ is a subset of $\widetilde{\mathcal{S}}$ as defined in (5). It then follows from Condition 1 that the inverse DFT of $c_{m}$ is supported on $\mathcal{S}$ as well. Consequently, we have

$$
\boldsymbol{c} \stackrel{\text { def }}{=}\left[\begin{array}{llll}
c_{0} & c_{1} & \ldots & c_{M-1}
\end{array}\right]^{T} \in \mathcal{R}(\boldsymbol{A}),
$$

where $\boldsymbol{A}$ is the frame analysis matrix we constructed before. A key observation is that we have access to the magnitude of the above frame coefficients, because

$$
\left|c_{m}\right|=\left|f_{m} f_{m-1}^{*}\right|=\left|f_{m}\right| \cdot\left|f_{m-1}\right| .
$$

Based on Condition 2, we can (almost always) obtain $\xi c$ from $\left\{\left|c_{m}\right|\right\}_{m}$, for some an unknown constant phase term $|\xi|=1$ (or a complex-conjugate version $\left.\xi c^{*}\right)$.

For simplicity, we assume that what we obtain is $\xi c$. (If we actually get the complex conjugate, the derivations will be similar and the final result differs only by a time-reversal.) For each $m$, we construct a $2 \times 2$ matrix

$$
\boldsymbol{M}_{m} \stackrel{\text { def }}{=}\left[\begin{array}{cc}
f_{m} f_{m}^{*} & f_{m} f_{m+1}^{*} \xi^{*} \\
f_{m+1} f_{m}^{*} \xi & f_{m+1} f_{m+1}^{*}
\end{array}\right]=\left[\begin{array}{c}
f_{m} \\
f_{m+1} \xi
\end{array}\right]\left[\begin{array}{ll}
f_{m}^{*} & f_{m+1}^{*} \xi^{*}
\end{array}\right] .
$$

Clearly, $\boldsymbol{M}_{m}$ is Hermitian and of rank one. Meanwhile, the factorization in (7) indicates that we can obtain the values of the Fourier coefficients $f_{m}, f_{m+1} \xi$ through an eigenvalue decomposition of $\boldsymbol{M}$. A catch here is that there exists a fundamental phase ambiguity in the factorization and we can only obtain $f_{m} \theta_{m}, f_{m+1} \xi \theta_{m}$ for some unknown phase term $\left|\theta_{m}\right|=1$. Although the matrices $\boldsymbol{M}_{m}$ for different $m$ will lead to different phase ambiguities, the overlapping nature of the data

$$
\begin{array}{llrl}
f_{1} \theta_{1} & f_{2} \xi \theta_{1} & & \\
& f_{2} \theta_{2} & f_{3} \xi \theta_{2} & \\
& & f_{3} \theta_{3} & f_{4} \xi \theta_{3}
\end{array}
$$

\footnotetext{
${ }^{1}$ To verify this property, we apply the polar identity of complex numbers to write $c_{m}=f_{m} f_{m-1}^{*}=\left(\left\|f_{m}+f_{m-1}\right\|^{2}-\left\|f_{m}-f_{m-1}\right\|^{2}+j \| f_{m}+\right.$ $\left.j f_{m-1}\left\|^{2}-j\right\| f_{m}-j f_{m-1} \|^{2}\right) / 4$. It follows that the inverse DFT of $c_{m}$ is a linear combination of four different autocorrelations sequences, all of which are supported within $\widetilde{S}$.
}

allows us to "align" all phase ambiguities into a single phase term $\theta_{1}$ and get

$$
\tilde{f}_{m}=f_{m} \xi^{m} \theta_{1}, \quad \text { for } 0 \leq m<M,
$$

with some unknown phase terms $\theta_{1}$ and $\xi$.

We can write the Fourier coefficients as $f_{m}=\sum_{k} c_{k} e^{-j 2 \pi n_{k} m / M}$, where $\left\{c_{k}\right\}$ and $\left\{n_{k}\right\}$ are the coefficients and locations of the nonzero elements of $x_{n}$. It follows that (8) can be rewritten as

$$
\tilde{f}_{m}=\sum_{k=1}^{K} \theta_{1} c_{k}\left(e^{-j 2 \pi n_{k} / M} \xi\right)^{m} .
$$

Note that the sum of exponents structure in (9) is a classical object in spectral analysis [7]. We can borrow tools (such as the annihilation filter method [7]) from that field to obtain the parameters $\left\{\theta_{1} c_{k}\right\}_{k}$ and $\left\{e^{-j 2 \pi n_{k} / M} \xi\right\}_{k}$ from $\widehat{f}_{m}$. Finally, after proper normalizations (to let $x_{0}>0$ ) and circular shiftings (to enforce the support on [0,N$1]$ ), we can obtain the coefficients $\left\{c_{k}\right\}$ and the locations $\left\{n_{k}\right\}$, and thus reconstruct the sparse signal $x_{n}$.

\section{RECONSTRUCTION ALGORITHM}

The proof presented in the previous section is constructive. Algorithm 1 summarizes the main steps of a reconstruction algorithm that can obtain a $K$-sparse signal from its autocorrelation.

\section{Algorithm 1 Sparse Spectral Factorization}

Input: The autocorrelation sequence $a_{n}$ of a $K$-sparse signal $x_{n}$.

Output: Reconstruct the sparse signal up to a time-reversal.

1: Obtain the support set $\mathcal{S}$ of the autocorrelation sequence $a_{n}$. Construct a matrix $\boldsymbol{A}$ by picking columns of an $M \times M$ DFT matrix according to $\mathcal{S}$.

2: Obtain $c_{m} \stackrel{\text { def }}{=} f_{m} f_{m-1}^{*}$ from the absolute values $\left|f_{m} f_{m-1}\right|$ up to some unknown phase $\xi$ or a possible complex conjugation (see Algorithm 2).

3: Building the $2 \times 2$ matrices $M_{m}$ as in (7), and factorize them to obtain $f_{m} \theta_{m}, f_{m+1} \xi \theta_{m}$.

4: From the overlapping portions of consecutive blocks, align all unknown phases to a single phase perturbation $\theta_{1}$.

5: Run the annihilation filter algorithm [7] on the sequence (9) to obtain the coefficients and the desired support set.

All the steps in Algorithm 1 are straightforward, except for step 2 , where we want to recover the sequence $c_{m}$ (up to an unknown phase and complex-conjugation) from their absolute values. In what follows, we present an iterative algorithm based on singular value projections to address this remaining challenge.

It follows from (6) that $\boldsymbol{c}=\boldsymbol{A} \boldsymbol{y}$ for some $\boldsymbol{y} \in \mathbb{R}^{L}$. Since $\boldsymbol{c}$ can be uniquely determined by $\boldsymbol{y}$, we focus on estimating $\boldsymbol{y}$ from the absolute values $|\boldsymbol{c}|$. Let $\boldsymbol{v}_{m}$ denote the $m$ th column of $\boldsymbol{A}^{*}$. We can easily verify that $c_{m}=\left\langle\boldsymbol{y}, \boldsymbol{v}_{m}\right\rangle$ and thus

$$
\left|c_{m}\right|=\left|\left\langle\boldsymbol{y}, \boldsymbol{v}_{m}\right\rangle\right| \text {. }
$$

Clearly, the mapping from $\boldsymbol{y}$ to $\left|\left\langle\boldsymbol{y}, \boldsymbol{v}_{m}\right\rangle\right|$ is nonlinear. However, we can convert it to a linear mapping by embedding the problem in a higher dimensional Hilbert space.

Definition 1 Let $\boldsymbol{S}_{L}$ denote the space of all $L \times L$ Hermitian matrices. For any $\boldsymbol{X}, \boldsymbol{Y} \in \boldsymbol{S}_{L}$, define their inner product as

$$
\langle X, Y\rangle \stackrel{\text { def }}{=} \operatorname{tr}\left(X Y^{*}\right),
$$

where $\operatorname{tr}(\cdot)$ denotes the trace of a matrix. 
One way to view the inner product defined in (11) is as follows: Let $\operatorname{vec}(\boldsymbol{X})$ and $\operatorname{vec}(\boldsymbol{Y})$ represent two vectors in $\mathbb{C}^{L^{2}}$ obtained by stacking the columns of $\boldsymbol{X}$ and $\boldsymbol{Y}$, respectively. Then

$$
\langle X, Y\rangle=(\operatorname{vec}(\boldsymbol{Y}))^{*} \operatorname{vec}(\boldsymbol{X})
$$

that is, the matrix inner product is equivalent to the usual Euclidian scalar product between $\operatorname{vec}(\boldsymbol{X})$ and $\operatorname{vec}(\boldsymbol{Y})$.

The following is a simple but key observation, whose proof can be found in e.g., [6]. It is remarkable in that it converts the original nonlinear condition in (10) into a set of linear constraints in the higher dimensional space $\boldsymbol{S}_{L}$.

Proposition 2 Denote by $\boldsymbol{Y}=\boldsymbol{y} \boldsymbol{y}^{*}$ and $\boldsymbol{V}_{m}=\boldsymbol{v}_{m} \boldsymbol{v}_{m}^{*}$ the rankone Hermitian matrices generated by $\boldsymbol{y}$ and $\boldsymbol{v}_{m}$, respectively. Then

$$
\left|\left\langle\boldsymbol{y}, \boldsymbol{v}_{m}\right\rangle\right|^{2}=\left\langle\boldsymbol{Y}, \boldsymbol{V}_{m}\right\rangle, \quad 0 \leq m<M .
$$

In what follows, we propose a simple and intuitive algorithm for reconstructing $\boldsymbol{y}$ from the magnitude information in (10). Our algorithm alternates between the following two constraints.

1. Linear constraint: It follows from (12) that each frame coefficient magnitude in (10) gives us a linear equation in the space of $\boldsymbol{S}_{L}$. In general, the set of $\boldsymbol{Y}$ satisfying all these linear constraints forms an affine subspace in $\boldsymbol{S}_{L}$.

2. Rank constraint: By construction, $\boldsymbol{Y}=\boldsymbol{y} \boldsymbol{y}^{*}$ is a positivedefinite matrix of rank-one. For an arbitrary Hermitian matrix $\boldsymbol{X}$, the best rank-one approximation of $\boldsymbol{X}$ can be computed by a singular value decomposition (SVD) of $\boldsymbol{X}$.

By iteratively enforcing the above two constraints, we can use Algorithm 2 to obtain $y$ from the magnitude information (10).

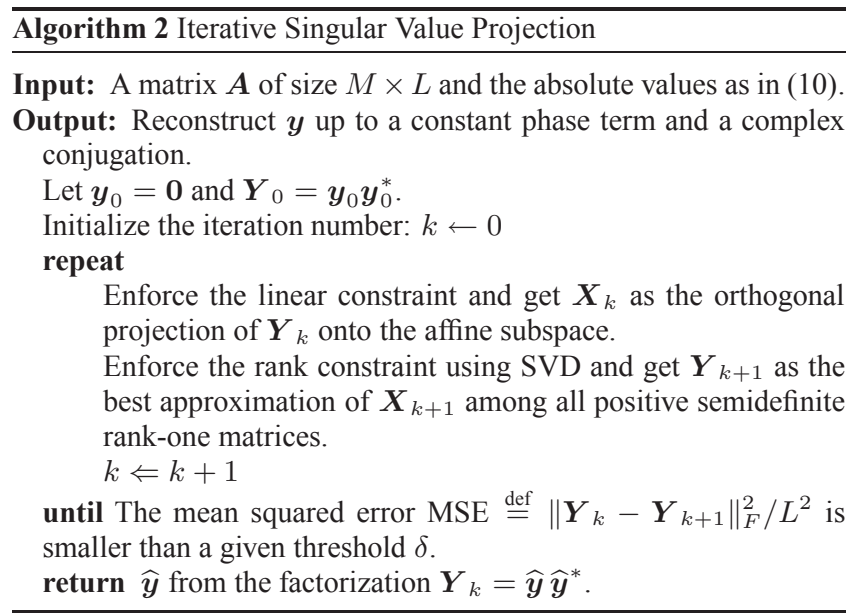

Example 2 Figure 2(a) shows the autocorrelation of an unknown $K$ sparse signal $x_{n}$. We do not need to know the exact value of $K$; only a rough upper bound will suffice. Applying Algorithm 1, we obtain a sparse signal $\widehat{x}_{n}$, shown in Figure 2(b). We can verify that it produces the same autocorrelation as the input. Note that another valid solution will be a time-reversed version, i.e., $x_{-n}$.

In step 2 of Algorithm 1, we employ the proposed iterative singular value projection scheme (Algorithm 2) to estimate $\left\{c_{m}\right\}$ from their absolute values. The algorithm converges within numerical precision after about $10^{4}$ iterations. The total running time on a computer with a $2.2 \mathrm{GHz} C P U$ is about 16 seconds.

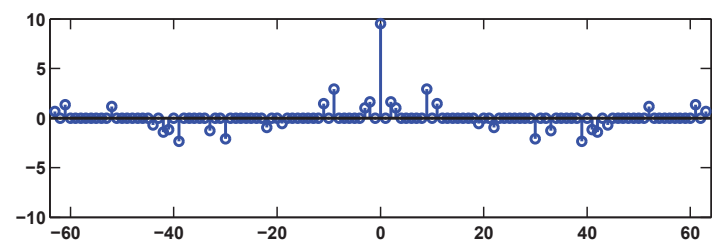

(a)

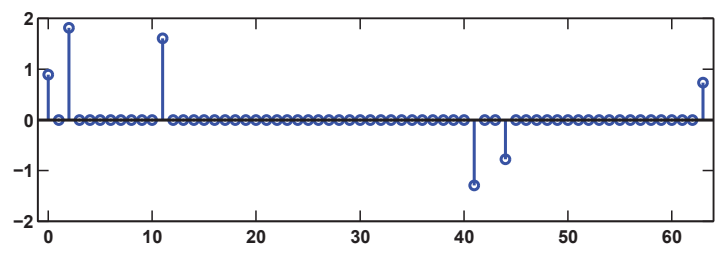

(b)

Fig. 2. (a) The autocorrelation of a $K$-sparse signal. (b) The reconstructed sparse signal $\widehat{x}_{n}$ obtained by the proposed algorithm.

Remark 2 Numerical experiments suggest that, when the unknown signal $x_{n}$ is "sufficiently sparse", Algorithm 2 always converges, despite the nonconvexity of the rank constraint. We leave the rigorous analysis of this convergence behavior to a future work.

\section{CONCLUSION}

We studied the problem of recovering a 1-D sparse signal from its autocorrelation. Our results show that there always exist sparse signals that cannot be determined by their autocorrelations, no matter how sparse they are. However, most signals with nonuniform support patterns are indeed recoverable. We present a sufficient condition for the reconstruction to be unique, based on which we proposed an efficient reconstruction algorithm using iterative singular value projections. Numerical examples verify the effectiveness of the proposed algorithm.

\section{REFERENCES}

[1] J. R. Fienup, "Phase retrieval algorithms: a comparison," Applied Optics, vol. 21, no. 15, pp. 2758-2769, 1982.

[2] A. H. Sayed and T. Kailath, "A survey of spectral factorization methods," Numer. Linear Algebra Appl., vol. 8, pp. 467-496, 2001.

[3] M. H. Hayes, J. S. Lim, and A. V. Oppenheim, "Signal reconstruction from phase or magnitude," IEEE Trans. Acoust., Speech, and Signal Process., vol. ASSP-28, no. 6, pp. 672-680, Dec. 1980

[4] T. R. Crimmins and J. R. Fienup, "Uniqueness of phase retrieval for functions with sufficiently disconnected support," J. Opt. Soc. Am., vol. 73, no. 2, pp. 218 - 221, Feb. 1983.

[5] W. L. Chan, M. L. Moravec, R. G. Baraniuk, and D. M. Mittleman, "Terahertz imaging with compressd sensing and phase retrieval," Opt. Lett., vol. 33, no. 9, pp. 974-976, May 2008.

[6] R. Balan, B. G. Bodmann, P. G. Casazza, and D. Edidin, "Painless reconstruction from magnitudes of frame coefficients," $J$. Fourier Anal. Appl., vol. 15, no. 4, pp. 488-501, 2009.

[7] P. Stoica and R. L. Moses, Introduction to spectral analysis, Prentice-Hall, Englewood Cliffs, NJ, 1997. 